\title{
Prior medication adherence of participants and non participants of a randomized controlled trial to improve patient adherence in cardiovascular risk management
}

\author{
A. Sieben ${ }^{1 *}$, S. J. H. Bredie ${ }^{2}$, J. C. H. B. M. Luijten ${ }^{3}$, C. J. H. M. van Laarhoven ${ }^{3}$, S. van Dulmen ${ }^{4,5,6}$ and \\ H. A. W. van Onzenoort ${ }^{7,8}$
}

\begin{abstract}
Background: Poor medication adherence is a major factor in the secondary prevention of cardiovascular diseases (CVD) and contributes to increased morbidity, mortality, and costs. Interventions for improving medication adherence may have limited effects as a consequence of self selection of already highly adherent participants into clinical trials.

Methods: In this retrospective cohort study, existing levels of medication adherence were examined in self-decided participants and non-participants prior to inclusion in a randomized controlled study (RCT), evaluating the effect of an intervention to improve adherence. In addition, the non-participants were further divided into 'responders' and 'non responders'. All individuals had manifest cardiovascular disease and completed a questionnaire with baseline characteristics, the Beliefs about Medicines Questionnaire (BMQ) and the Modified Morisky Scale ${ }^{\oplus}\left(M^{-} S^{\oplus}\right)$ as part of a regular screening program. A logistic regression was conducted to examine the relationship between study participation willingness, adherence level and the beliefs about medication.
\end{abstract}

Results: According to the $\mathrm{MMS}^{\circledR}$ the adherence level was comparable in all groups. In both (non)-participants groups, 36\% was classified as high adherent; $46 \%$ participants versus $44 \%$ non-participants were classified as medium adherent and $19 \%$ of the participants versus $20 \%$ of the non-participants were low adherent $(p=0.91$. The necessity concern differential (NCD) from the BMQ was 3.8 for participants and 3.4 for non-participants $(p=0.32$ ).

Conclusion: This study shows that adherence to medication and beliefs about medication do not differ between participants and non-participants before consenting to participate in an RCT. The study design seems not to have led to greater adherence in the study group.

Keywords: Randomized controlled trials, Informed consent, Participation, Selection bias, Adherence

\footnotetext{
* Correspondence: angelien.sieben@radboudumc.nl

${ }^{1}$ Department of Surgery, Division of Vascular Surgery, Radboud university medical center, Geert Grooteplein 10, Postbus 9101, 6500 HB Nijmegen, the Netherlands

Full list of author information is available at the end of the article
}

(c) The Author(s). 2019 Open Access This article is distributed under the terms of the Creative Commons Attribution 4.0 International License (http://creativecommons.org/licenses/by/4.0/), which permits unrestricted use, distribution, and reproduction in any medium, provided you give appropriate credit to the original author(s) and the source, provide a link to the Creative Commons license, and indicate if changes were made. The Creative Commons Public Domain Dedication waiver (http://creativecommons.org/publicdomain/zero/1.0/) applies to the data made available in this article, unless otherwise stated. 


\section{Background}

Cardiovascular risk reduction is predominantly based on lipid and blood pressure lowering treatment, inhibition of platelet aggregation, smoking cessation and control of obesity [1]. A limitation in lowering cardiovascular risk is poor adherence to prescribed medication [2] which may consequently can lead to increased morbidity, mortality and costs [3-6]. Nevertheless, a recent review with mostly cohort studies, showed that only $60 \%$ of people who use cardiovascular medication, were adherent to their cardiovascular medication [7]. In view of that there is a need for interventions to improve medication adherence in this population. Although there is a considerable amount of research in the field of interventions to improve medication adherence in cardiovascular patients, they often show only small effects [8]. It is suggested that patient recruitment methods in randomized controlled trials (RCT) to improve patient adherence to medication may influence outcome $[8,9]$. An important observation is that patients participating in RCTs generally have higher adherence rates at baseline than could be expected based on observational studies [10-14]. It is conceivable that the informed consent procedure results in a selection of patients with higher adherence rates [12]. Willingness to participate is positively influenced by patients' engagement with their medical condition, high level of education and the influence of an important person $[11,15]$. These characteristics are also considered as positive determinants for medication adherence [16]. Although a recent review showed that the inclusion of non adherent patients was the single feature significantly associated with effective adherence interventions, most studies seem to include patients because they are willing to participate not because they are poor adherent [11]. It is suggested that patients participating in these RCTs already have a pre-existing high adherence level at baseline [10, 12-14]. Selection of participants with high levels of adherence at baseline, makes it difficult to show an improving intervention effect (ceiling effect) [8]. More understanding about the medication adherence of participants as well as non-participants before the start of these RCT's may contribute to a better understanding of why so many studies show no improvement in medication adherence. One possible explanatory determinant for (non) adherent behaviour is medication beliefs. Personal beliefs about need for treatment (necessity beliefs) and concerns about several potential adverse consequences (concern beliefs) could explain a large part of (non) adherent behaviour [16-18]. If patients perceive that the need for medication outweighs the concerns, they are more likely to be adherent to their medication(s) [19].

\section{Methods}

Aim

The aim of this study is to explore possible differences in adherence to existing prescribed medication in cardiovascular patients who did or did not consent to participate in an RCT which expressly explored the effects of an intervention to improve adherence. We hypothesized that patients who are willing to participate in a clinical trial are more likely to be medication adherent and have more 'necessity beliefs' about their medication compared to patients who are not willing to participate.

\section{Study design and setting}

In this retrospective cohort study we included patients who participated or declined participation in the (MIRROR) trial (a Multifaceted nurse -and web-based Intervention for impRoving adheRence to treatment in patients with cardiOvasculaR disease) [20]. In brief, the MIRROR trial was a prospective, randomized controlled trial in which patients aged $\geq 18$ years and diagnosed with a manifest cardiovascular disease (i.e. acute coronary syndrome, peripheral arterial disease or stroke/Transient Ischemic Attack (TIA)) after providing written informed consent, were included. The MIRROR trial aimed to study the effect of different adherence enhancing strategies on cardiovascular medication adherence. Within this context, patients were randomized to usual care, an e-health intervention, and an e-health intervention combined with motivational technique consultations.

\section{Participants}

All patients referred to the Radboud University Medical Center with a new diagnosis of acute coronary syndrome, myocardial infarction, peripheral arterial disease, an aneurysm of the aorta or TIA or stroke over the prior 6 weeks were included into the hospital CVD screening program. This screening program aims to identify cardiovascular risk factors and consists of screening for lifestyle (smoking, diet and exercise), medication adherence by the self reported questionnaires Modified Morisky Scale $^{\circ}\left(\mathrm{MMS}^{\circ}\right)$ and the Beliefs about Medication Questionnaire (BMQ), blood lipid levels, blood pressure, waist circumference, body mass index (BMI), glucose blood levels and a family history of cardiovascular diseases. If indicated, preventive therapies (medication and lifestyle interventions) are initiated and followed over time [1]. All patients referred to this screening program were asked to participate in the MIRROR- trial. 'Participants' were patients who were willing to participate in the intervention study and 'non-participants' were patients who declined informed consent for the MIRROR trial. Because adherence to medication may also differ between responders and non- responders to surveys, with responders having higher adherence levels [21] we 
divided the group of non-participants further. Retrospectively of the MIRROR trial, a letter was sent to all non-participants for a different study not subject to this paper. For this study, a signed informed consent was requested from the non-participants. Non-responders were patients who did not sign this letter. Responders were patients who signed the informed consent letter. We aimed to explore if the non-responding subgroup of the non-participants differed from the responders with respect to their level of medication adherence on the basis of prior $\mathrm{MMS}^{\circ}$ from the screening program.

\section{Ethical approval}

The Ethical Committee waived the need for a formal informed consent for this study. The study was conducted according to the good clinical practice protocol and we used usual care data considering the research question of this study. Data was anonymized according to the research protocols of the Ethical Committee.

\section{Outcomes}

Participation or declining to participate to the RCT was the independent variable in this study. Adherence to medication and the beliefs about medication the dependent variables. Adherence to cardiovascular medication was calculated by the $\mathrm{MMS}^{\odot}$ [22-24]. It consists of eight items aimed at measuring adherence. Each item accounts for 0 or 1 in the case questions are answered by no or yes respectively. Consequently, total $\mathrm{MMS}^{\circ}$ scores range between 0 and 8 . These scores were divided into three levels of adherence: low adherence (sum score $<6$ ), medium adherence (sum score 6 to $<8$ ) and high adherence (sum score of 8) [25]. To evaluate patients' beliefs and perceptions about their medication, BMQ [26] was used. This validated questionnaire provides information about the beliefs, perceived necessity and concerns the patient has regarding their illness and prescribed medication. There are five statements regarding "necessity beliefs" and five regarding "concern beliefs". Patients indicated their degree of agreement with each individual statement about the use of their medicines on a five-point Likert scale. Thus, total scores for the necessity and concerns scales could range from 5 to 25. The necessity- concerns differential (NCD) was then calculated as the difference between necessity and concerns scores and had a possible range of -20 to 20 $[19,27]$. To differentiate between patients on the basis of their beliefs about the necessity of their medication and their concerns about taking medication, the total necessity and concern scores [5-25] were split at midpoint (thus 5-12 was considered as low and $13 \mathrm{t} / \mathrm{m} 25$ was considered as high). Patients were then classified into four different categories: accepting (high necessity and low concerns), ambivalent (high necessity and high concerns), skeptical (high concerns and low necessity) and indifferent (low concerns and low necessity) [2830].

From all patients the type of CVD (acute coronary syndrome, myocardial infarction, peripheral arterial disease, an aneurysm of the aorta or TIA) was recorded. Also, the following baseline and clinical characteristics were collected: age, sex, level of education, employment status, the country of origin and the type of cardiovascular medication used.

\section{Data collection and timeline}

Data were derived from the screening program. Data were registered in a secure website which could only be accessed by nurses involved in the screening program. Within, on average, six weeks after the CVD-event, baseline characteristics and the questionnaires were collected for all patients as part of the screening program.

\section{Statistical analyses}

Data were analyzed and evaluated using SPSS version 22. Descriptive statistics (mean, median, standard deviation) were used for all variables. A Mann-Whitney Test was used to compare groups (participants and non-participants) with the non parametric outcome, the $\mathrm{MMS}^{\circ}$. Confounders were explored by performing a logistic linear test of all the characteristics in the case the groups significantly differed (including the NCD). The same Mann-Whitney test was performed to compare the NCD between the groups. A logistic regression was used to explore differences between (non) participants and the four belief groups. The same statistical analyses were performed for the responders and non-responders. A Kruskal-Wallis test was performed to explore the relationship between the NCD and the $\mathrm{MMS}^{\circ}$ for all groups.

\section{Results}

In total, 900 patients with a new cardiovascular event between October 2011 and October 2013 were eligible for participation into the MIRROR trial. Of these, 419 agreed and 481 refused participation. Of all the non-participants who received a letter for another study, 220 did not respond. Consequently, 261 non-participants were classified as responders (Fig. 1).

\section{Patient characteristics}

The total cohort (participants and non-participants) had a mean age of 62 years and was predominantly male (67\%). Participants significantly differed from non-participants with respect to age (61 years versus 63 years, $p=0.001$ ), male sex $(71 \%$ versus $58 \%, p=0.001)$, systolic blood pressure $(136 \mathrm{mmHg}$ versus $142 \mathrm{mmHg}, p=0.001)$. Participants were more frequently diagnosed with an acute coronary syndrome ( $36 \%$ versus $16 \%, p<0.001])$, were 


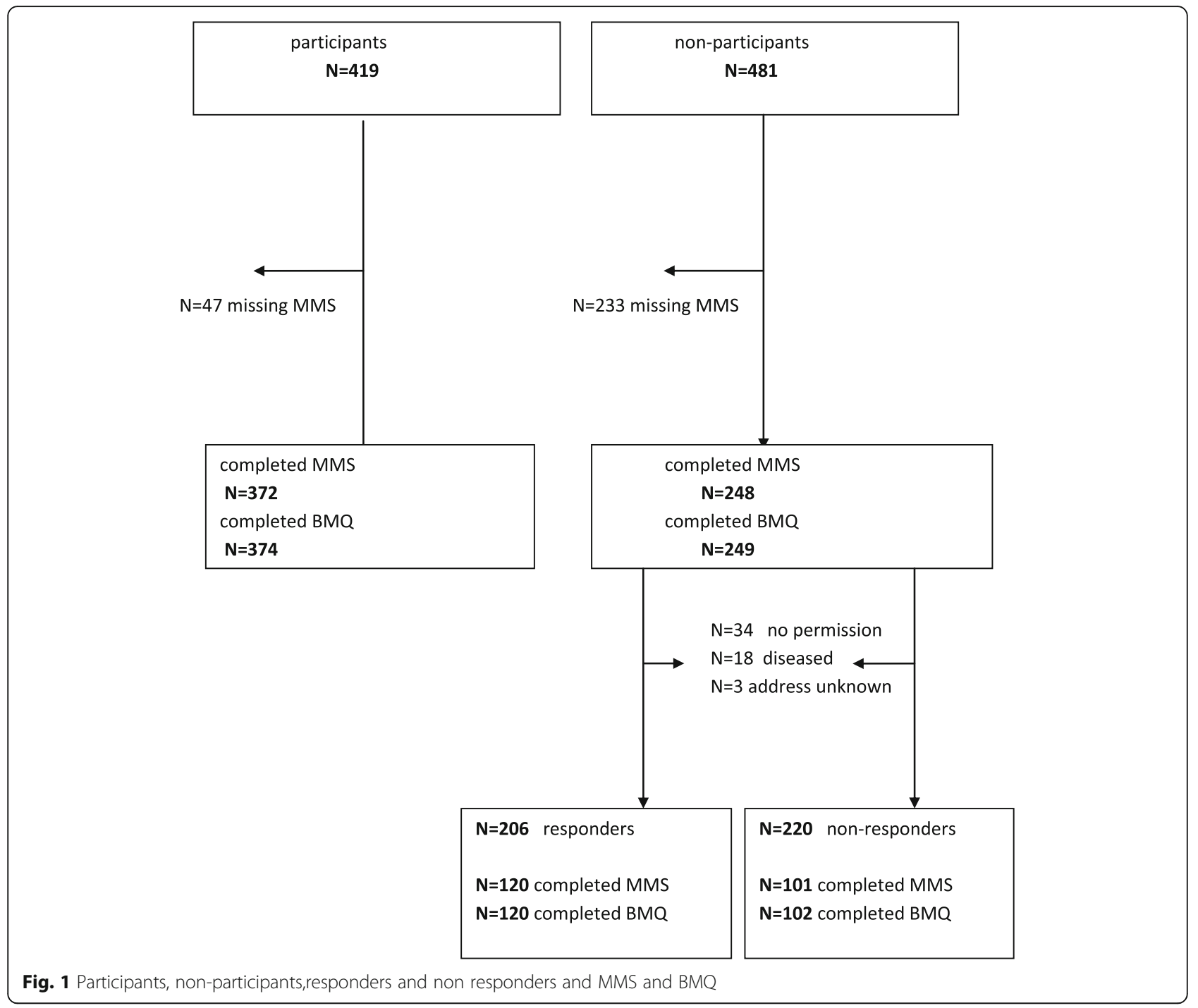

using more beta blocking agents or agents acting on the renin-angiotensin system (58\% versus $46 \% p=0.001$ and $59 \%$ versus $44 \% p=0.001$, respectively), and were using more lipid lowering medication ( $94 \%$ versus $82 \%, p<0.001)$. Among the non-participants, responders were older (64 versus 60 years, $p=0.002$ ) and used more agents acting on the renin-angiotensin system ( $48 \%$ versus $37 \%, p=0.02$ ) than non-responders (Tables 1 and 2).

\section{Medication adherence}

We did not observe differences in adherence measured by the $\mathrm{MMS}^{\circ}$ between both groups $(p=0.99)$. According to the $\mathrm{MMS}^{\bullet} 19 \%$ of the participants was classified as low adherers compared to $20 \%$ in the non-participants group. Forty-six percent of the participants and $44 \%$ of the non-participants were classified as medium adherers, whereas 36 and $37 \%$ were classified as high adherers, respectively. There were no differences in adherence according to the $\mathrm{MMS}^{\bullet}$ between responders and non-responders $(p=0.47)$. In both the responders and non-responders group, 36\% scored high on adherence. Sixteen percent of the responders were low adherent compared to $24 \%$ in the non-responder group. Forty-eight percent of the responder group scored a medium adherence and $41 \%$ of the non-responders. Compared to study participation all characteristics that significantly differed between both groups were separately analyzed by a logistic regression analyses. None of the variables significantly influenced the association between study participation and adherence according to the $\mathrm{MMS}^{\circ}$ (Tables 3 and 4).

\section{Beliefs about medication}

Based on the BMQ the necessity concerns differential (NCD) was 3.8 among participants compared to 3.4 among non-participants $(p=0.13)$. Of all the participating and non-participating patients $26 \%$ were in the accepting group, $67 \%$ in the ambivalent group, $3 \%$ in the skeptical and $4 \%$ in the indifferent group. No differences 
Table 1 Differences in patient characteristics between participants and non participants in a RCT-trial on adherence

\begin{tabular}{|c|c|c|c|}
\hline & Participants [ $n=419]$ & Non-participants $[n=481]$ & $P$-value \\
\hline Age $[$ mean, \pm SD) & $60.5[ \pm 10]$ & $63[11]$ & 0.001 \\
\hline \multicolumn{4}{|l|}{ Gender (N [\%]) } \\
\hline Male & $296[7]$ & $279[58]$ & \multirow[t]{2}{*}{$<0.001$} \\
\hline Female & $123[29$ & $202[42]$ & \\
\hline \multicolumn{3}{|l|}{ Education level (N[\%]) } & \multirow[t]{4}{*}{$<0.001$} \\
\hline Primary & $66[18]$ & $141[31]$ & \\
\hline Secondary & 185 [49] & $180[39]$ & \\
\hline University & 124 [33] & $140[30]$ & \\
\hline \multicolumn{3}{|l|}{ Labour (N[\%]) } & \multirow[t]{4}{*}{0.08} \\
\hline Paid labour & 137 [37] & $123[27]$ & \\
\hline Unemployed & 98 [26.3] & $138[30]$ & \\
\hline Retired & 138 [37] & 199 [43.3] & \\
\hline \multicolumn{3}{|l|}{ Country of origin is the Netherland (N[\%]) } & \multirow[t]{3}{*}{0.66} \\
\hline Yes & $327[90]$ & $398[86]$ & \\
\hline No & $37[10]$ & $64[14]$ & \\
\hline \multicolumn{3}{|l|}{ Reason referral (N[\%]) } & \multirow[t]{4}{*}{$<0.001$} \\
\hline acute coronary syndrome & $150[36]$ & $79[16]$ & \\
\hline peripheral arterial disease & $71[17]$ & $101[21]$ & \\
\hline troke/TIA & $198[47]$ & $301[63]$ & \\
\hline \multicolumn{3}{|l|}{ Blood pressure $(m m H g ;$ mean \pm SD) } & $<0.001$ \\
\hline Systolic & $136[ \pm 18]$ & $142[ \pm 20]$ & \multirow[t]{2}{*}{0.23} \\
\hline Diastolic & $77[ \pm 11]$ & $78[ \pm 11]$ & \\
\hline Body Mass Index (mean \pm SD) & $27[ \pm 4]$ & $26[ \pm 4]$ & 0.30 \\
\hline \multicolumn{4}{|l|}{ Waist (mean \pm SD) } \\
\hline Male & $99.5[ \pm 9]$ & $98.4[ \pm 12]$ & 0.10 \\
\hline Female & $92[ \pm 14]$ & $90[ \pm 13]$ & 0.07 \\
\hline \multicolumn{4}{|l|}{ Lipids (mmol/ltr, mean SD) } \\
\hline Totaal cholesterol & $4.5[ \pm 1.1]$ & $4.6[ \pm 1]$ & 0.7 \\
\hline Triglyceriden & $1.8[ \pm 1]$ & $1.7[ \pm 1]$ & 0.01 \\
\hline $\mathrm{HDL}$ & $1.2[ \pm 0.3]$ & $1.2[ \pm 0.3]$ & 0.002 \\
\hline LDL & $2.5[ \pm 0.9]$ & $2.6[ \pm 0.9]$ & 0.66 \\
\hline \multicolumn{4}{|l|}{ Medication (N [\%]) } \\
\hline Antithrombotic agents [ATC B01] & $404[98]$ & $461[98]$ & 0.78 \\
\hline Diuretics [ATC C03] & $109[26]$ & $135[29]$ & 0.44 \\
\hline Beta Blocking agents [ATC C07] & $239[58]$ & $218[46]$ & 0.001 \\
\hline Calcium channel blockers [ATCC08] & $65[16]$ & $72[15]$ & 0.86 \\
\hline Agents acting on [..] system [ATC C09] & 244 [59] & $206[44]$ & 0.001 \\
\hline Lipid modifying agents [ATC C10] & 387 [94] & $384[82]$ & $<0.001$ \\
\hline
\end{tabular}

were observed between the two groups $(p=0.23)$. The mean score of the NCD in the responders and non-responders groups was 3.6 and 3.1 respectively ( $p=$ 0.21 ). Among the non-responders $24 \%$ were in the accepting group, $61 \%$ in the ambivalent group, $6 \%$ in the skeptical group and $9 \%$ in the indifferent group. For the responders this was $27,72,2$ and $0 \%$ respectively. Differences between both groups were statistically significant $(p<0.01)$. Logistic regression analysis on NCD did not significantly influence the association between study participation and adherence according to the $\mathrm{MMS}^{\circ}$. 
Table 2 Differences in patient characteristics between responders and non-responders

\begin{tabular}{|c|c|c|c|}
\hline & responder $[n=206]$ & Non-responder [ $n=220]$ & $P$-value \\
\hline Age (mean \pm SD) & $64[10]$ & $60[12]$ & 0.002 \\
\hline \multicolumn{4}{|l|}{ Gender (N [\%]) } \\
\hline Male & $120[58]$ & $129[59]$ & \multirow[t]{2}{*}{0.93} \\
\hline Female & $86[42]$ & $91[41]$ & \\
\hline \multicolumn{4}{|l|}{ Education level (N[\%]) } \\
\hline Primary & $52[26]$ & $67[32]$ & \multirow[t]{3}{*}{0.43} \\
\hline Secondary & $80[41]$ & $81[39]$ & \\
\hline University & 66 [33] & $62[29]$ & \\
\hline \multicolumn{4}{|l|}{ Labour (N [\%]) } \\
\hline Paid labour & $49[25]$ & 68 [33] & \multirow[t]{3}{*}{0.08} \\
\hline Unemployed & $55[28]$ & $64[31]$ & \\
\hline Retired & $94[47]$ & 77 [36] & \\
\hline \multicolumn{4}{|l|}{ Country of origin is the Netherlands(N[\%]) } \\
\hline Yes & $174[88]$ & $181[86]$ & \multirow[t]{2}{*}{0.62} \\
\hline No & $24[12]$ & $29[14]$ & \\
\hline \multicolumn{3}{|l|}{ Reason referral (N[\%]) } & \multirow[t]{4}{*}{0.89} \\
\hline acute coronary syndrome & $34[16]$ & $37[17]$ & \\
\hline peripheral arterial disease & $47[23]$ & $46[21]$ & \\
\hline stroke/TIA & $125[61]$ & $137[62]$ & \\
\hline \multicolumn{4}{|l|}{ Blood pressure $(m m H g ;$ mean \pm SD) } \\
\hline Systolic & $140[ \pm 19]$ & $142[ \pm 20]$ & 0.30 \\
\hline Diastolic & $78[ \pm 11]$ & $79[ \pm 10]$ & 0.23 \\
\hline Body Mass Index (mean \pm SD) & $26[ \pm 4]$ & $26[ \pm 4]$ & 0.22 \\
\hline \multicolumn{4}{|l|}{ Waist (mean \pm SD) } \\
\hline Male & $97[ \pm 11]$ & $99[ \pm 12]$ & 0.16 \\
\hline Female & $91[ \pm 13]$ & $89[ \pm 13]$ & 0.36 \\
\hline \multicolumn{4}{|l|}{ Lipids (mmol/ltr; mean \pm SD) } \\
\hline Totaal cholesterol & $4.6[ \pm 1]$ & $4.6[ \pm 0.9]$ & 0.73 \\
\hline Triglyceriden & $1.7[ \pm 1]$ & $1.7[ \pm 0.9]$ & 0.01 \\
\hline $\mathrm{HDL}$ & $1.3[ \pm 0.3]$ & $1.2[ \pm 0.3]$ & 0.06 \\
\hline LDL & $2.5[ \pm 0.9]$ & $2.6[ \pm 0.9]$ & 0.78 \\
\hline \multicolumn{4}{|l|}{ Medication(N [\%]) } \\
\hline Antithrombotic agents [ATC B01] & $196[97]$ & $213[98]$ & 0.27 \\
\hline Diuretics [ATC C03] & $62[31]$ & $56[26]$ & 0.28 \\
\hline Beta Blocking agents [ATC C07] & $93[46]$ & $96[44]$ & 0.74 \\
\hline Calcium channel blockers [ATCC08] & $30[15]$ & $34[16]$ & 0.80 \\
\hline Agents acting on [..] system [ATC C09] & 98 [48] & $80[37]$ & 0.02 \\
\hline Lipid modifying agents [ATC C10] & $166[82]$ & $173[80]$ & 0.59 \\
\hline
\end{tabular}

\section{Discussion}

To our knowledge this is the first study exploring the differences in medication adherence in patients who did or did not consent to participate in an RCT evaluating the effect of an intervention to improve medication adherence. Our study showed that patients willing to participate in an RCT evaluating the effect of an intervention to improve medication adherence, have a comparable adherence level to patients who declined participation. Even by further exploring the non-participant group in 
Table 3 Differences participants and non-participants in adherence and beliefs about medication

\begin{tabular}{llll}
\hline & Totaal & Non-participants & Participants \\
\hline Adherence according to the MMS N [\%] & & & \\
Low adherence & $119[19]$ & $49[20]$ & $70[19]$ \\
Medium adherence & $279[45]$ & $109[44]$ & $170[46]$ \\
High adherence & $222[36]$ & $90[36]$ & $132[35]$ \\
NCD mean [SD] & $3.65[ \pm 4.8]$ & $3.4[ \pm 5]$ & $3.8[ \pm 4.9]$ \\
Belief Groups [N\%] & & & \\
Accepting & $160[26]$ & $61[24]$ & $100[27]$ \\
Ambivalent & $418[67]$ & $165[67]$ & $255[68]$ \\
Sceptical & $19[3]$ & $10[4]$ & $9[2]$ \\
Indifferent & $23[4]$ & $13[10]$ & $10[3]$ \\
\hline
\end{tabular}

responders and non-responders, we did not observe differences in adherence between the groups. Consequently, the results of this study suggest that a population representative in adherence level participated in an RCT evaluating the effect of an intervention to improve medication adherence.

Previous studies suggested that patients not participating in RCTs to improve medication adherence have a different pre-existing adherence level from patients who participate [10-14]. This was supported by the observed differences in adherence levels between these RCTs and observational studies [10-14]. Typically, adherence levels among patients in RCTs were higher than in observational studies. Although not different among participants and non-participants, adherence in this study was also high. An explanation for the high adherence rate in both groups could be that we started inclusion for the RCT within six weeks after the cardiovascular event. For cardiovascular patients who just had an event, the need for adherent behaviour is emerging [31, 32]. Yet, as the event fades and symptoms subside, adherence levels can also decline [33]. Research with a long follow up is needed to establish if there will be a difference in adherence between participants and non-participants over time.

In all groups, we observed significant differences in patient characteristics. Compared to non-participants, participants were younger and more were highly educated. This was also observed among responders and non-responders. These are known as prognostic characteristics for patients who are willing to participate in a clinical trial [15] and for a high adherence level [34, 35]. Although the relationship between socio-demographic variables and adherence is mainly weak and inconsistent $[34,36,37]$ it was expected that these characteristics could have been an explanation for the assumed higher adherence rates in the participant groups. However, we could not support this hypothesis. Also, next to the high adherence rate, a high mean NCD score was present in all groups. This only confirmed the adherent behaviour in both groups [19]. It is also congruent with earlier studies showing that medication beliefs can be a more powerful predictor of medication adherence than clinical and socio-demographic factors [19, 38]. However, we did not observe a relationship between NCD and trial

Table 4 Differences responders and non-responders in adherence and belief about medication

\begin{tabular}{llll}
\hline & Totaal & Non-responders & Responders \\
\hline Adherence according to the MMS N [\%] & & & \\
Low adherence & $43[20]$ & $24[24]$ & $19[16]$ \\
Medium adherence & $99[45]$ & $41[40]$ & $58[48]$ \\
High adherence & $79[36]$ & $36[36]$ & $43[36]$ \\
NCD mean [SD] & $3.6[ \pm 4.9]$ & $3.1[ \pm 5]$ & $4[ \pm 4.9]$ \\
Belief Groups [N\%] & & & $32[27]$ \\
Accepting & $56[25]$ & $24[24]$ & $86[72]$ \\
Ambivalent & $148[67]$ & $62[61]$ & $2[2]$ \\
Sceptical & $8[4]$ & $6[6]$ & 0.17 \\
Indifferent & $10[4]$ & $10[9]$ & 0.001 \\
\hline
\end{tabular}


participation. We did observe a significant difference in the beliefs about medication groups in the (non) responder groups. More patients of the non-responder group were also in the indifferent and skeptical group. Non-responders of surveys are known for more negative evaluations of healthcare [39]. This could be associated with higher concern beliefs in medication as these are partly influenced by the prescriber-patient relationship in healthcare [40]. The number of patients who differed in these groups was very small and the NCD did not differ. More research is needed to draw any conclusions on this point.

This study had some limitations. We had to deal with missing data especially in the self reported questionnaires $\mathrm{BMQ}$ and $\mathrm{MMS}^{\circ}$. There were fewer missing in the participants group compared to the non-participants group. The questionnaires were just implemented in the screening program. As the questionnaires were also part of the MIRROR trial, more attention could have been paid to participants for documenting these questionnaires. So there were more patients in the non-participant group who did not fill out the MMS ${ }^{\circ}$. These patients could very well be non-adherent [21]. There are different methods available to measure adherence. Each method has advantages and disadvantages. The $\mathrm{MMS}^{\circ}[22,24,41]$ is a validated questionnaire that can be easily applied to large populations. As $\mathrm{MMS}^{\circ}$ is a subjective measure, adherence levels may be higher than what is expected in real life. Refill data from the out-patient pharmacy on the other hand has been used extensively to provide insight into drug acquisition and dispensing [42]. However, to use the pharmacy refill data we need an informed consent from patients. This study however used data from patients collected only in standard care because we wanted to include patients who declined participation in a RCT. Other methods, such as MEMS or pill count, seem to influence patient's behavior through direct confrontation. Moreover, application of MEMS is relatively expensive, especially when applied in standard care [42]. The BMQ was used because, to our knowledge, is the only validated questionnaire that evaluates patients' beliefs, necessity and concerns the patient has according to his illness and prescribed medication. This in contrast to other validated adherence questionnaires that measure specific medication-taking behavior of patients [26, 43]. The high NCD score confirmed the prediction of adherent behaviour in both groups $[19,38]$.

\section{Conclusion}

This study showed no differences in medication adherence between participants and non-participants prior to the inclusion of the MIRROR trial. A representative group seems to have participated in this randomized controlled trial designed to improve medication adherence [20].

\section{Abbreviations}

BMI: Body mass index; BMQ: Beliefs about Medicines Questionnaire; CVD: Cardiovascular diseases; MIRROR trial: A Multifaceted nurse -and webbased Intervention for impRoving adheRence to treatment in patients with cardiOvasculaR disease; MMS : Modified Morisky Scale ${ }^{\oplus}$; NCD: Necessity concern differential; RCT: Randomized controlled study; TIA: Transient Ischemic Attack

\section{Acknowledgements \\ Not applicable.}

Funding

There was no funding for this study.

Availability of data and materials

The datasets generated and analyzed during this study are not publicly available due to the Dutch privacy laws. But are available from the corresponding author on reasonable request.

\section{Authors' contributions}

AS, SB, HvO have agreed to be accountable for all aspects of the work in ensuring that questions related to the accuracy or integrity of any part of the work are appropriately investigated and resolved. AS and JL have made substantial contributions to conception and design, or acquisition of data, or analysis and interpretation of data. SVD and KVL have been involved in drafting the manuscript or revising it critically for important intellectual content. All authors have given final approval of the version to be published. Each author has participated sufficiently in the work to take public responsibility for appropriate portions of the content.

\section{Ethics approval}

The Ethical Committee (the human related research committee of the Arnhem-Nijmegen region) waived the need for a formal informed consent for this study. The study was conducted according to the good clinical practice protocol and we used usual care data considering the research question of this study. Data was anonymised according to the research protocols of the Ethical Committee.

\section{Consent for publication}

Not applicable.

\section{Competing interests}

The authors declare that they have no competing interest.

\section{Publisher's Note}

Springer Nature remains neutral with regard to jurisdictional claims in published maps and institutional affiliations.

\section{Author details}

'Department of Surgery, Division of Vascular Surgery, Radboud university medical center, Geert Grooteplein 10, Postbus 9101, 6500 HB Nijmegen, the Netherlands. ${ }^{2}$ Department of General Internal Medicine, Division of Vascular Medicine, Radboud university medical center, Nijmegen, the Netherlands. ${ }^{3}$ Department of General Surgery, Radboud university medical center, Nijmegen, the Netherlands. ${ }^{4}$ Department of Primary and Community Care, Radboud university medical center, Radboud Institute for Health Sciences, Nijmegen, the Netherlands. ${ }^{5}$ NIVEL (Netherlands institute for health services research), Utrecht, the Netherlands. ${ }^{6}$ Faculty of Health and Social Sciences, University of South-Eastern Norway, Drammen, Norway. ${ }^{7}$ Department of Clinical Pharmacy, Amphia Hospital, Breda, the Netherlands. ${ }^{8}$ Department of Clinical Pharmacy and Toxicology, Maastricht University Medical Center+, Maastricht, the Netherlands.

Received: 16 October 2018 Accepted: 29 April 2019

Published online: 09 May 2019

\section{References}

1. De Backer G, Ambrosioni E, Borch-Johnsen K, Brotons C, Cifkova R, Dallongeville J, et al. European guidelines on cardiovascular disease prevention in clinical practice; third joint task force of European and other societies on cardiovascular disease prevention in clinical practice 
(constituted by representatives of eight societies and by invited experts). Eur J Cardiovasc Prev Rehabil. 2003;10(4):S1-S10.

2. Munger MA, Van Tassell BW, LaFleur J. Medication nonadherence: an unrecognized cardiovascular risk factor. MedGenMed. 2007;9(3):58.

3. Rasmussen JN, Chong A, Alter DA. Relationship between adherence to evidence-based pharmacotherapy and long-term mortality after acute myocardial infarction. JAMA. 2007;297(2):177-86.

4. Simpson RJ Jr, Mendys P. The effects of adherence and persistence on clinical outcomes in patients treated with statins: a systematic review. J Clin Lipidol. 2010;4(6):462-71.

5. De Vera MA, Bhole V, Burns LC, Lacaille D. Impact of statin adherence on cardiovascular disease and mortality outcomes: a systematic review. Br J Clin Pharmacol. 2014;78(4):684-98.

6. Bansilal S, Castellano JM, Garrido E, Wei HG, Freeman A, Spettell C, et al. Assessing the impact of medication adherence on long-term cardiovascular outcomes. J Am Coll Cardiol. 2016;68(8):789-801.

7. Chowdhury R, Khan H, Heydon E, Shroufi A, Fahimi S, Moore C, et al. Adherence to cardiovascular therapy: a meta-analysis of prevalence and clinical consequences. Eur Heart J. 2013;34(38):2940-8.

8. Nieuwlaat R, Wilczynski N, Navarro T, Hobson N, Jeffery R, Keepanasseril A, et al. Interventions for enhancing medication adherence. Cochrane Database Syst Rev. 2014;11:Cd000011.

9. Jeffery RA, Navarro T, Wilczynski NL, Iserman EC, Keepanasseril A, Sivaramalingam B, et al. Adherence measurement and patient recruitment methods are poor in intervention trials to improve patient adherence. J Clin Epidemiol. 2014;67(10):1076-82.

10. van Onzenoort HA, Menger FE, Neef C, Verberk WJ, Kroon AA, de Leeuw PW, et al. Participation in a clinical trial enhances adherence and persistence to treatment: a retrospective cohort study. Hypertension. 2011;58(4):573-8.

11. Allemann SS, Nieuwlaat R, Navarro T, Haynes B, Hersberger KE, Arnet I. Congruence between patient characteristics and interventions may partly explain medication adherence intervention effectiveness: an analysis of 190 randomized controlled trials from a Cochrane systematic review. J Clin Epidemiol. 2017:91:70-9.

12. Wetzels GE, Nelemans P, Schouten JS, Prins MH. Facts and fiction of poor compliance as a cause of inadequate blood pressure control: a systematic review. J Hypertens. 2004;22(10):1849-55.

13. Horne R, Clatworthy J, Hankins M. High adherence and concordance within a clinical trial of antihypertensives. Chronic IIIn. 2010;6(4):243-51.

14. Atar D, Ong S, Lansberg PJ. Expanding the evidence base: comparing randomized controlled trials and observational studies of statins. Am J Ther. 2015;22(5):e141-50.

15. Ross S, Grant A, Counsell C, Gillespie W, Russell I, Prescott R. Barriers to participation in randomised controlled trials: a systematic review. J Clin Epidemiol. 1999;52(12):1143-56.

16. Horne RWJ, Barber N, et al. Concordance, adherence and compliance in medicine taking. London: national Co-ordinating Centre for NHS Service and Organisation R\&D (NCCSDO); 2006.

17. Hugtenburg JG, Timmers L, Elders PJ, Vervloet M, van Dijk L. Definitions, variants, and causes of nonadherence with medication: a challenge for tailored interventions. Patient Prefer Adherence. 2013;7:675-82.

18. Kardas P, Lewek P, Matyjaszczyk M. Determinants of patient adherence: a review of systematic reviews. Front Pharmacol. 2013;4:91.

19. Horne R, Weinman J. Patients' beliefs about prescribed medicines and their role in adherence to treatment in chronic physical illness. J Psychosom Res. 1999;47(6):555-67.

20. Sieben A, van Onzenoort HA, van Laarhoven KJ, Bredie SJ. A multifaceted nurse- and web-based intervention for improving adherence to treatment in patients with cardiovascular disease: rationale and design of the MIRROR trial. JMIR Res Protoc. 2016;5(3):e187.

21. Gadkari AS, Pedan A, Gowda N, McHorney CA. Survey nonresponders to a medication-beliefs survey have worse adherence and persistence to chronic medications compared with survey responders. Med Care. 2011;49(10):956-61.

22. Krousel-Wood M, Islam T, Webber LS, Re RN, Morisky DE, Muntner P. New medication adherence scale versus pharmacy fill rates in seniors with hypertension. Am J Manag Care. 2009;15(1):59-66.

23. Krousel-Wood MA, Muntner P, Islam T, Morisky DE, Webber LS. Barriers to and determinants of medication adherence in hypertension management: perspective of the cohort study of medication adherence among older adults. Med Clin North Am. 2009;93(3):753-69.
24. Morisky DE, Ang A, Krousel-Wood M, Ward HJ. Predictive validity of a medication adherence measure in an outpatient setting. The Journal of Clinical Hypertension. 2008;10(5):348-54.

25. Korb-Savoldelli V, Gillaizeau F, Pouchot J, Lenain E, Postel-Vinay N, Plouin P$F$, et al. Validation of a French version of the 8-item Morisky medication adherence scale in hypertensive adults. The Journal of Clinical Hypertension. 2012;14(7):429-34.

26. Horne R, Weinman J, Hankins M. The beliefs about medicines questionnaire: the development and evaluation of a new method for assessing the cognitive representation of medication. Psychol Health. 1999;14(1):1-24.

27. Clifford S, Barber N, Horne R. Understanding different beliefs held by adherers, unintentional nonadherers, and intentional nonadherers: application of the necessity-concerns framework. J Psychosom Res. 2008;64(1):41-6.

28. Menckeberg TT, Bouvy ML, Bracke M, Kaptein AA, Leufkens HG, Raaijmakers JAM, et al. Beliefs about medicines predict refill adherence to inhaled corticosteroids. J Psychosom Res. 2008;64(1):47-54.

29. Aikens JE, Nease DE Jr, Nau DP, Klinkman MS, Schwenk TL. Adherence to maintenance-phase antidepressant medication as a function of patient beliefs about medication. Ann Fam Med. 2005;3(1):23-30.

30. Clatworthy J, Bowskill R, Parham R, Rank T, Scott J, Horne R. Understanding medication non-adherence in bipolar disorders using a necessity-concerns framework. J Affect Disord. 2009;1 16(1-2):51-5.

31. Jackevicius CA, Li P, Tu JV. Prevalence, predictors, and outcomes of primary nonadherence after acute myocardial infarction. Circulation. 2008:117(8):1028-36

32. Miller NH. Adherence behavior in the prevention and treatment of cardiovascular disease. Journal of Cardiopulmonary Rehabilitation and Prevention. 2012;32(2):63-70 https://doi.org/10.1097/HCR.0b013e318235c729.

33. Ho PM, Bryson CL, Rumsfeld JS. Medication adherence: its importance in cardiovascular outcomes. Circulation. 2009;119(23):3028-35.

34. Brown MT, Bussell JK. Medication adherence: WHO cares? Mayo Clin Proc. 2011;86(4):304-14.

35. Mauskop A, Borden W. Predictors of statin adherence. Curr Cardiol Rep. 2011;13(6):553-8.

36. Schedlbauer A, Davies P, Fahey T. Interventions to improve adherence to lipid lowering medication. Cochrane Database Syst Rev. 2010;3:CD004371.

37. Miller NH, Hill M, Kottke T, Ockene IS, Compliance FtEPo. The multilevel compliance challenge: recommendations for a call to action: a statement for healthcare professionals. Circulation. 1997;95(4):1085-90.

38. Foot H, La Caze A, Gujral G, Cottrell N. The necessity-concerns framework predicts adherence to medication in multiple illness conditions: a metaanalysis. Patient Educ Couns. 2016;99(5):706-17.

39. Perneger TV, Chamot E, Bovier PA. Nonresponse bias in a survey of patient perceptions of hospital care. Med Care. 2005;43(4):374-80.

40. Horne R, Chapman SC, Parham R, Freemantle N, Forbes A, Cooper V. Understanding patients' adherence-related beliefs about medicines prescribed for long-term conditions: a meta-analytic review of the necessity-concerns framework. PLoS One. 2013;8(12):e80633.

41. Morisky DE, DiMatteo MR. Improving the measurement of self-reported medication nonadherence: response to authors. J Clin Epidemiol. 2011;64(3):255-7 discussion 8-63.

42. Osterberg L, Blaschke T. Adherence to medication. N Engl J Med. 2005;353(5):487-97.

43. Horne R, Buick D, Fisher M, Leake H, Cooper V, Weinman J. Doubts about necessity and concerns about adverse effects: identifying the types of beliefs that are associated with non-adherence to HAART. Int J STD AIDS. 2004;15(1):38-44. 